International Journal of Pure and Applied Mathematics

Volume 88 No. 1 2013, 91-103

ISSN: 1311-8080 (printed version); ISSN: 1314-3395 (on-line version)

url: http://www.ijpam.eu

doi: http://dx.doi.org/10.12732/ijpam.v88i1.7

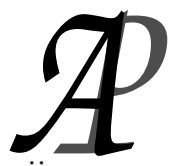

ijpam.eu

\title{
ROTATION-INVARIANT $L^{p}$-FUNCTIONS
}

\author{
Areerak Chaiworn $^{1 \S}$, Pheerachate Bunpatcharajarurn ${ }^{2}$ \\ ${ }^{1}$ Department of Mathematics \\ Faculty of Science \\ Burapha University \\ Chonburi, 20131, THAILAND \\ ${ }^{2}$ Centre of Excellence in Mathematics \\ CHE, Si Ayutthaya Rd., Bangkok 10400, THAILAND
}

\begin{abstract}
This paper show that the space of rotation-invariant $L^{p}$-functions with respect to a Gaussian measure can established as an even $L^{p}$-space on $\mathbb{R}$ with respect to some non-Gaussian measure. The space of holomorphic rotation-invariant $L^{p}$-functions with respect to a complex Gaussian measure can established as a holomorphic even $L^{p}$-space on $\mathbb{C}$ with respect to some non-complex Gaussian measure. We give a condition for a rotation-invariant function which the image of the Segal-Bargmann transform to be in the space of holomorphic rotation-invariant $L^{q}$-functions with respect to a complex Gaussian measure.
\end{abstract}

AMS Subject Classification: Segal-Bargmann transform, Segal-Bargmann space, rotation-invariance

\section{Introduction}

The Segal-Bargmann transform is an integral transform $B$ which maps $L^{2}\left(\mathbb{R}^{d}, \rho\right)$, the set of all functions on $\mathbb{R}^{d}$ that are square integrable with respect to the real

Received: July 11, 2013

(C) 2013 Academic Publications, Ltd.

$\S$ Correspondence author url: www.acadpubl.eu 
Gaussian measure $\rho(x) d x=(2 \pi)^{-d / 2} e^{-x^{2} / 2} d x$, onto $\mathcal{H} L^{2}\left(\mathbb{C}^{d}, \mu\right)$, the set of all holomorphic functions on $\mathbb{C}^{d}$ that are square integrable with respect to the complex Gaussian measure $\mu(z) d z=(\pi)^{-d} e^{-|z|^{2}} d z$. The transform $B$ is given by this formula

$$
(B f)(z)=\int_{\mathbb{R}^{d}} f(x) \frac{e^{-(z-x)^{2} / 2}}{(2 \pi)^{d / 2}} d x
$$

for all $f \in L^{2}\left(\mathbb{R}^{d}, \rho\right)$ and $z \in \mathbb{C}^{d}$. Here we use notation $x^{2}=x_{1}^{2}+x_{2}^{2}+\cdots+x_{d}^{2}$ for $x=\left(x_{1}, x_{2}, \ldots, x_{n}\right) \in \mathbb{R}^{n}$. The space $\mathcal{H} L^{2}\left(\mathbb{C}^{d}, \mu\right)$ is also called the SegalBargmann space. See [1], [2], [3], [4], [5], [6], [8] for details about the importance of this space.

In [9], [10] and [11], Chaiworn and Lewkeeratiyutkul consider the subspace of the Segal-Bargmann which is invariant under the action of the complex special orthogoal group. They construct the non-Gaussian measure $\gamma$ on $\mathbb{R}$ which the space $L^{2}\left(\mathbb{R}^{d}, \rho\right)^{S O(d)}$ is unitarily equivalent to the space $L^{2}(\mathbb{R}, \gamma)^{e}$. In the same way they also construct the non-Gaussian measure $\lambda$ on $\mathbb{C}$ which the Segal-Bargmann space is unitarily equivalent to the Hilbert space $\mathcal{H} L^{2}(\mathbb{C}, \lambda)^{e}$.

In this paper we will denote $L^{p}\left(\mathbb{R}^{d}, \rho\right)^{S O(d)}$ by the set of all $S O(d)$-invariant $L^{p}$ functions with respect to a Gaussian measure and $\mathcal{H} L^{p}\left(\mathbb{C}^{d}, \mu\right)^{S O(d, \mathbb{C})}$ by the space of all $S O(d, \mathbb{C})$-invariant holomorphic $L^{p}$-functions on $\mathbb{C}^{d}$ with respect to a complex Gaussian measure. The $S O(d)$-invariance of real valued $f$ is determined by it value on $(x, 0, \ldots, 0) \cong \mathbb{R}$ and it is an even function. Thus the space $L^{p}\left(\mathbb{R}^{d}, \rho\right)^{S O(d)}$ and $L^{p}(\mathbb{R}, \gamma)^{e}$ are isometrically isomorphic. Similarly a complex $S O(d, \mathbb{C})$-invariant function $F$ is determined by its values on $(z, 0, \ldots, 0) \cong \mathbb{C}$ and it is a complex even function. Then we have, the space $\mathcal{H} L^{p}\left(\mathbb{C}^{d}, \mu\right) S O(d, \mathbb{C})$ and $\mathcal{H} L^{p}(\mathbb{C}, \lambda)^{e}$ are isometrically isomorphic. We consider the Segal-Bargmann transform applied to the function on $L^{p}\left(\mathbb{R}^{d}, \rho\right)^{S O(d)}$ with $1<p<\infty$. In [7], Hall obtain that if $F=B(f)$ for some $f \in L^{p}\left(\mathbb{R}^{d}, \rho\right)$, then

$$
\mid F(x+i y) \leq C e^{y^{2} / 2} e^{x^{2} /(p-1)} .
$$

In this paper we will use this bound and Theorem 7 of [7] to give a sufficient

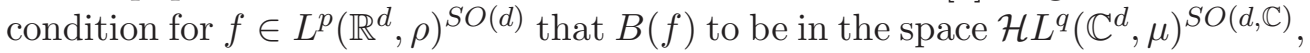
when $\frac{1}{q}+\frac{1}{p}=1,0<q \leq 2$ and $p \geq \frac{q}{2}+1$.

\section{Rotation-Invariant Function Spaces}

Denote by $S O(d)$ the set of $d \times d$ real orthogonal matrices with determinant one and by $S O(d, \mathbb{C})$ the set of $d \times d$ complex orthogonal matrices with determinant one. 
Definition 1. Let $F$ be a function on $\mathbb{F}^{d}$ where $\mathbb{F}$ is $\mathbb{C}$ or $\mathbb{R}$ and let $G$ be a group of $d \times d$ matrices. We say that $F$ is $G$-invariant if

$$
F(A x)=F(x) \text { for all } A \in G \text { and all } x \in \mathbb{F}^{d} .
$$

Note that if $F$ is an $S O(d)$-invariant holomorphic function on $\mathbb{C}^{d}$, then by analytic continuation it is $S O(d, \mathbb{C})$-invariant.

Denote by $\mathcal{B}_{d}$ the Borel $\sigma$-algebra in $\mathbb{R}^{d}$ and by $\mathcal{B}$ the Borel $\sigma$-algebra in $\mathbb{R}$. Define the maps $\Psi_{i}:\left(\mathbb{R}^{d}, \mathcal{B}_{d}, \rho\right) \rightarrow(\mathbb{R}, \mathcal{B}), i=1,2$ by

$$
\Psi_{1}(x)=|x| \text { and } \Psi_{2}(x)=-|x|
$$

for all $x \in \mathbb{R}^{d}$. For each $E \in \mathcal{B}$ let

$$
\gamma_{i}(E)=\rho\left(\Psi_{i}^{-1}(E)\right),
$$

and let $\gamma=\left(\gamma_{1}+\gamma_{2}\right) / 2$. It is easy to check that $\gamma$ is a Borel measure on $\mathbb{R}$ and for any measurable function $g$ and any $E \in \mathcal{B}$

$$
\int_{E} g d \gamma=\frac{1}{2} \int_{\Psi_{1}^{-1}(E)} g \circ \Psi_{1} d \rho+\frac{1}{2} \int_{\Psi_{2}^{-1}(E)} g \circ \Psi_{2} d \rho .
$$

Denote by $\mathcal{F}\left(\mathbb{R}^{d}\right)^{S O(d)}$ the set of all $S O(d)$-invariant functions on $\mathbb{R}^{d}$ and $\mathcal{F}(\mathbb{R})^{e}$ the set of all even functions on $\mathbb{R}$. We write

$$
\begin{aligned}
L^{p}\left(\mathbb{R}^{d}, \rho\right)^{S O(d)} & =\mathcal{F}\left(\mathbb{R}^{d}\right)^{S O(d)} \cap L^{p}\left(\mathbb{R}^{d}, \rho\right) \\
L^{p}(\mathbb{R}, \gamma)^{e} & =\mathcal{F}(\mathbb{R})^{e} \cap L^{p}(\mathbb{R}, \gamma) .
\end{aligned}
$$

Form [10] we know that the measure $\gamma$ is absolutely continuous with respect to Lebesgue measure on $\mathbb{R}$ with density given by

$$
\Delta(s)=\frac{\sigma\left(S^{d-1}\right)}{(2 \pi)^{d / 2}}|s|^{d-1} e^{-s^{2} / 2} .
$$

where $S^{d-1}$ is a unit sphere on $\mathbb{R}^{d}$ and $\sigma$ is the surface measure on $S^{d-1}$.

Theorem 2. [10] For any $d \geq 2$, the map $\Phi: \mathcal{F}\left(\mathbb{R}^{d}\right)^{S O(d)} \rightarrow \mathcal{F}(\mathbb{R})^{e}$ defined by

$$
\Phi(G)(s)=G(s, 0, \ldots, 0)
$$

for all $G \in \mathcal{F}\left(\mathbb{R}^{d}\right)^{S O(d)}$ and all $s \in \mathbb{R}$, is a linear isomorphism whose inverse is given by

$$
\Psi(g)(x)=g(\sqrt{(x, x)})=g(|x|)
$$

for all $g \in \mathcal{F}(\mathbb{R})^{e}$ and all $x=\left(x_{1}, x_{2}, \ldots, x_{d}\right) \in \mathbb{R}^{d}$. 
Proof. See Theorem 1 in [10].

Theorem 3. The spaces $L^{p}\left(\mathbb{R}^{d}, \rho\right)^{S O(d)}$ and $L^{p}(\mathbb{R}, \gamma)^{e}$ are isometrically isomorphic.

Proof. From Theorem 2 we have that the function

$$
\Psi: \mathcal{F}(\mathbb{R})^{e} \rightarrow \mathcal{F}\left(\mathbb{R}^{d}\right)^{S O(d)}
$$

is a linear isomorphism. We consider the restriction of $\Psi$ to the space $L^{p}(\mathbb{R}, \gamma)^{e}$. Let $g \in \mathcal{F}(\mathbb{R})^{e}$ and $G \in \mathcal{F}\left(\mathbb{R}^{d}\right)^{S O(d)}$ be such that $G=\Psi(g)$. Thus

$$
\begin{aligned}
\int_{\mathbb{R}}|g|^{p} d \gamma & =\frac{1}{2} \int_{\Psi_{1}^{-1}(\mathbb{R})}\left|g \circ \Psi_{1}(x)\right|^{p} \rho(x) d x+\frac{1}{2} \int_{\Psi_{2}^{-1}(\mathbb{R})}\left|g \circ \Psi_{2}(x)\right|^{p} \rho(x) d x \\
& =\int_{\mathbb{R}^{d}}|g(|x|)|^{p} \rho(x) d x \\
& =\int_{\mathbb{R}^{d}}|\Psi(g)(x)|^{p} \rho(x) d x \\
& =\int_{\mathbb{R}^{d}}|G(x)|^{p} \rho(x) d x .
\end{aligned}
$$

So $\|g\|_{L^{p}(\mathbb{R}, \gamma)}=\|G\|_{L^{p}\left(\mathbb{R}^{d}, \rho\right)}$. Hence, $G \in L^{p}\left(\mathbb{R}^{d}, \rho\right)^{S O(d)}$ if and only if $g \in L^{p}(\mathbb{R}$, $\gamma)^{e}$. This shows that $\Psi$ is an isometry map from $L^{p}(\mathbb{R}, \gamma)^{e}$ onto $L^{p}\left(\mathbb{R}^{d}, \rho\right)^{S O(d)}$.

In the same way denote by $\mathcal{H}\left(\mathbb{C}^{d}\right)^{S O(d, \mathbb{C})}$ the set of all $S O(d, \mathbb{C})$-invariant holomorphic functions on $\mathbb{C}^{d}$, and $\mathcal{H}(\mathbb{C})^{e}$ the set of all holomorphic even functions on $\mathbb{C}$. Therefore we have the following theorem for complex case.

Theorem 4. For any $d \geq 2$, the map $\phi: \mathcal{H}\left(\mathbb{C}^{d}\right)^{S O(d, \mathbb{C})} \rightarrow \mathcal{H}(\mathbb{C})^{e}$ defined by

$$
\phi(f)(\xi)=f(\xi, 0, \ldots, 0)
$$

for any $f \in \mathcal{H}\left(\mathbb{C}^{d}\right)^{S O(d, \mathbb{C})}$ and any $\xi \in \mathbb{C}$, is a linear isomorphism whose inverse is given by

$$
\psi(g)(z)=g(\sqrt{(z, z)})
$$

for any $g \in \mathcal{H}(\mathbb{C})^{e}$ and any $z \in \mathbb{C}^{d}$ where $(z, z)=z_{1}^{2}+z_{2}^{2}+\cdots+z_{d}^{2}$.

Note that since $g$ is even, the value of $\phi(g)(z)$ is independent of the choice of square root of $(z, z)$.

In the same way denote by $\mathcal{B}\left(\mathbb{C}^{d}\right)$ the Borel $\sigma$-algebra in $\mathbb{C}^{d}$ and by $\mathcal{B}(\mathbb{C})$ the Borel $\sigma$-algebra in $\mathbb{C}$ and define $\Phi_{i}:\left(\mathbb{C}^{d}, \mathcal{B}\left(\mathbb{C}^{d}\right), \mu\right) \rightarrow(\mathbb{C}, \mathcal{B}(\mathbb{C})), i=1,2$ to 
be the branch of $\sqrt{(z, z)}$ with a smaller and larger argument respectively and for each $E \in \mathcal{B}(\mathbb{C})$ define

$$
\lambda_{i}(E)=\mu\left(\Phi_{i}^{-1}(E)\right)
$$

and let $\lambda=\left(\lambda_{1}+\lambda_{2}\right) / 2$.

Define

$$
\mathcal{H} L^{p}\left(\mathbb{C}^{d}, \mu\right)^{S O(d, \mathbb{C})}=\mathcal{H}\left(\mathbb{C}^{d}\right)^{S O(d, \mathbb{C})} \cap L^{p}\left(\mathbb{C}^{d}, \mu\right)
$$

and

$$
\mathcal{H} L^{p}(\mathbb{C}, \lambda)^{e}=\mathcal{H}(\mathbb{C})^{e} \cap L^{p}(\mathbb{C}, \lambda) .
$$

We now have the following theorem whose proof is similar to that of Theorem 3 .

Theorem 5. The spaces $\mathcal{H} L^{p}\left(\mathbb{C}^{d}, \mu\right)^{S O(d, \mathbb{C})}$ and $\mathcal{H} L^{p}(\mathbb{C}, \lambda)^{e}$ are isometrically isomorphic.

Theorem 6. [9] The measure $\lambda$ is absolutely continuous with respect to Lebesgue measure on $\mathbb{C}$ with density given by

$$
\Lambda(w)=\frac{|w|^{2 d-2}}{(\pi)^{d}} \int_{S_{1}} e^{-|w z|^{2}} d \alpha(z) .
$$

Proof. See Proposition 6 in [9].

In [9], we established that the density $\Lambda$ is equivalent to the function $\beta(w)=$ $|w|^{d-1} \frac{e^{-|w|^{2}}}{\pi}$ for all $w \in \mathbb{C}$ bounded away from zero.

Lemma 7. Let $p$ be a number with $1<p<\infty$. The norms $\|\cdot\|_{L^{p}(\mathbb{C}, \beta)}$ and $\|\cdot\|_{L^{p}(\mathbb{C}, \lambda)}$ are equivalent, i.e., there are constants $k, K>0$, depending on $d$, such that

$$
k\|f\|_{L^{p}(\mathbb{C}, \beta)} \leq\|f\|_{L^{p}(\mathbb{C}, \lambda)} \leq K\|f\|_{L^{p}(\mathbb{C}, \beta)},
$$

for all $f \in \mathcal{H} L^{p}(\mathbb{C}, \lambda)$.

Proof. First, we will show that there is a constant $D>0$, depending on $d$, such that

$$
\|f\|_{L^{p}(\mathbb{C}, \beta)}^{p} \leq D\|f\|_{L^{p}(\mathbb{C}-\mathbb{D}, \lambda)}^{p}
$$

for any $f \in \mathcal{H} L^{p}(\mathbb{C}, \lambda)$, where $\mathbb{D}=\{w \in \mathbb{C}:|w| \leq 1\}$. 
Let $w \in \mathbb{D}$. Denote by $A(w)$ the annulus $\{z \in \mathbb{C}|2 \leq| z-w \mid \leq 3\}$. For any $v \in A(w)$, we use the polar coordinates with the origin at $w$ so that $v-w=r e^{i \theta}$. If $f \in \mathcal{H} L^{p}(\mathbb{C}, \lambda)$, then we expand $f$ as a power series around $v=w$ :

$$
f(v)=f(w)+\sum_{n=1}^{\infty} a_{n}(v-w)^{n}
$$

Hence,

$$
\int_{A(w)} f(v) d v=f(w)(9 \pi-4 \pi)+\sum_{n=1}^{\infty} \int_{2}^{3} \int_{0}^{2 \pi} a_{n} r^{n} e^{i n \theta} d \theta d r=5 \pi f(w) .
$$

Therefore

$$
\begin{aligned}
f(w) & =\frac{1}{5 \pi} \int_{A(w)} f(v) d v \\
& =\frac{1}{5 \pi} \int_{\mathbb{C}-\mathbb{D}} \chi_{A(w)}(v) \frac{1}{\Lambda(v)} f(v) \Lambda(v) d v
\end{aligned}
$$

By Hölder's inequality we have that

$$
|f(w)| \leq \frac{1}{5 \pi}\left\|\chi_{A(w)} \frac{1}{\Lambda}\right\|_{L^{q}(\mathbb{C}-\mathbb{D}, \lambda)}\|f\|_{L^{p}(\mathbb{C}-\mathbb{D}, \lambda)}
$$

provided $\frac{1}{p}+\frac{1}{q}=1$. Since $\Lambda$ is strictly positive and continuous on $A(w), \frac{1}{\Lambda}$ is bounded on $A(w)$. Thus $\left\|\chi_{A(w)} \frac{1}{\Lambda}\right\|_{L^{q}(\mathbb{C}-\mathbb{D}, \lambda)}$ is finite. However, for each $w \in \mathbb{D}$,

$$
\frac{1}{5 \pi}\left\|\chi_{A(w)} \frac{1}{\Lambda}\right\|_{L^{q}(\mathbb{C}-\mathbb{D}, \lambda)} \leq \frac{1}{5 \pi}\left\|\chi_{A^{*}} \frac{1}{\Lambda}\right\|_{L^{q}(\mathbb{C}-\mathbb{D}, \lambda)}:=c<\infty
$$

where $A^{*}=\{z \in \mathbb{C}: 1<|z|<4\}$, which contains each $A(w), w \in \mathbb{D}$. Hence for any $w \in \mathbb{D}$

$$
|f(w)| \leq c\|f\|_{L^{p}(\mathbb{C}-\mathbb{D}, \lambda)}
$$

Since the density $\Lambda$ is equivalent to the function $\beta(w)=|w|^{d-1} \frac{e^{-|w|^{2}}}{\pi}$ for all $w \in \mathbb{C}$ bounded away from zero, we have that

$$
\begin{aligned}
\int_{\mathbb{C}}|f(w)|^{p} \beta(w) d w & =\int_{\mathbb{D}}|f(w)|^{p} \beta(w) d w+\int_{\mathbb{C}-\mathbb{D}}|f(w)|^{p} \beta(w) d w \\
& \leq c^{2}\|f\|_{L^{p}(\mathbb{C}-\mathbb{D}, \lambda)}^{p} \int_{\mathbb{D}} \beta(w) d w+\frac{1}{m}\|f\|_{L^{p}(\mathbb{C}-\mathbb{D}, \lambda)}^{p}
\end{aligned}
$$




$$
\leq D\|f\|_{L^{p}(\mathbb{C}-\mathbb{D}, \lambda)}^{p}
$$

for some constant $D>0$ depending on $d$. This give the first inequality in (3). The second inequality in (3) can be proved in the same way.

\section{The Segal-Bargmann Transform of Rotation-Invariant $L^{p}$ Functions}

For any $f \in L^{p}\left(\mathbb{R}^{d}, \rho\right)$ with $1<p<\infty$. Define the Segal-Bargmann transform $B(f)$ of $f$ by

$$
(B f)(z)=\int_{\mathbb{R}^{d}} f(x) \frac{e^{-(z-x)^{2} / 2}}{(2 \pi)^{d / 2}} d x, z \in \mathbb{C}^{d} .
$$

By Morera's Theorem, we have that $B(f) \in \mathcal{H}\left(\mathbb{C}^{d}\right)$. If $f \in L^{p}\left(\mathbb{R}^{d}, \rho\right)$ and $B(f)=0$, then $f=0$ i.e., $B$ is $1-1$.

Theorem 8. A function $f \in L^{p}\left(\mathbb{R}^{d}, \rho\right)$ is $S O(d)$-invariant if and only if $B(f) \in \mathcal{H}\left(\mathbb{C}^{d}\right)^{S O(d, \mathbb{C})}$.

Proof. Let $f \in L^{p}\left(\mathbb{R}^{d}, \rho_{t}\right)$ and $F=B(f)$.

$(\Rightarrow)$ Assume that $f$ is $S O(d)$-invariant. Define a bilinear form $(\cdot, \cdot)$ on $\mathbb{F}^{d}$ by

$$
(x, y)=x_{1} y_{1}+x_{2} y_{2}+\cdots+x_{d} y_{d}
$$

for all $x, y \in \mathbb{F}^{d}$. Then the elements of $S O(d)$ and $S O(d, \mathbb{C})$ preserve the bilinear form on $\mathbb{R}^{d}$ and $\mathbb{C}^{d}$ respectively. If $A \in S O(d)$ and $z \in \mathbb{C}^{d}$, then

$$
\begin{aligned}
F(A z) & =\frac{1}{(2 \pi)^{d / 2}} \int_{\mathbb{R}^{d}} f(x) e^{-(A z-x)^{2} / 2} d x \\
& =\frac{1}{(2 \pi)^{d / 2}} \int_{\mathbb{R}^{d}} f(x) e^{-\left(z-A^{-1} x\right)^{2} / 2} d x \\
& =\frac{1}{(2 \pi)^{d / 2}} \int_{\mathbb{R}^{d}} f(A x) e^{-(z-x)^{2} / 2} d(A x) \\
& =\frac{1}{(2 \pi)^{d / 2}} \int_{\mathbb{R}^{d}} f(x) e^{-(z-x)^{2} / 2} d x \\
& =F(z) .
\end{aligned}
$$


We use the fact that $\operatorname{det}(A)=1$ in the change of variables above. Hence, $F$ is $S O(d)$-invariant. By analytic continuation, $F$ is $S O(d, \mathbb{C})$-invariant.

$(\Leftarrow)$ Assume that $F$ is $S O(d, \mathbb{C})$-invariant. Let $A \in S O(d)$ and

$$
g(x)=f(A x) \quad \text { for all } x \in \mathbb{R}^{d} .
$$

Then $g \in L^{p}\left(\mathbb{R}^{d}, \rho\right)$ and for any $z \in \mathbb{C}^{d}$

$$
\begin{aligned}
B(g)(z) & =\frac{1}{(2 \pi)^{d / 2}} \int_{\mathbb{R}^{d}} g(x) e^{-(z-x)^{2} / 2} d x \\
& =\frac{1}{(2 \pi)^{d / 2}} \int_{\mathbb{R}^{d}} f(A x) e^{-(z-x)^{2} / 2} d x \\
& =\frac{1}{(2 \pi)^{d / 2}} \int_{\mathbb{R}^{d}} f(x) e^{-\left(z-A^{-1} x\right)^{2} / 2} d\left(A^{-1} x\right) \\
& =\frac{1}{(2 \pi)^{d / 2}} \int_{\mathbb{R}^{d}} f(x) e^{-(A z-x)^{2} / 2} d x \\
& =F(A z)=F(z)=B(f)(z) .
\end{aligned}
$$

Since $B$ is $1-1$, we must have $g=f$. Hence $f$ is $S O(d)$-invariant.

Define the operator $\mathcal{A}: \mathcal{H}\left(\mathbb{C}^{d}\right)^{S O(d, \mathbb{C})} \rightarrow \mathcal{H}\left(\mathbb{C}^{d}\right)^{S O}(d, \mathbb{C})$ by

$$
(\mathcal{A F})(z)=(z, z) F(z)
$$

for all $z \in \mathbb{C}^{d}$ where $(z, z)=z_{1}^{2}+\cdots+z_{d}^{2}$.

Lemma 9. For any $F \in \mathcal{H}\left(C^{d}\right)^{S O(d, \mathbb{C})}, f=\phi(F)$ and any $n \in \mathbb{N}$,

$$
\left\|\mathcal{A}^{n}(F)\right\|_{L^{p}\left(\mathbb{C}^{d}, \mu\right)}=\left\|w^{2 n} f\right\|_{L^{p}(\mathbb{C}, \lambda)} .
$$

Proof. Let $F \in \mathcal{H}\left(C^{d}\right)^{S O(d, \mathbb{C})}$ and $f=\phi(F)$. Then for all $w \in \mathbb{C}, f(w)=$ $F(w, 0, \ldots, 0)$ and for all $n \in \mathbb{N}$

$$
\begin{aligned}
\left\|\mathcal{A}^{n}(F)\right\|_{L^{p}\left(\mathbb{C}^{d}, \mu\right)}^{p} & =\int_{\mathbb{C}^{d}}\left|\left(\mathcal{A}^{n} F\right)(z)\right|^{p} \mu(z) d z \\
& =\int_{\mathbb{C}}\left|\phi\left(\mathcal{A}^{n} F\right)(w)\right|^{p} \lambda(w) d w \\
& =\int_{\mathbb{C}}\left|\left(\mathcal{A}^{n} F\right)(w, 0, \ldots, 0)\right|^{p} \lambda(w) d w
\end{aligned}
$$




$$
\begin{aligned}
& =\int_{\mathbb{C}}\left|w^{2 n} F(w, 0, \ldots, 0)\right|^{p} \lambda(w) d w \\
& =\int_{\mathbb{C}}\left|w^{2 n} f(w)\right|^{p} \lambda(w) d w \\
& =\left\|w^{2 n}(f)\right\|_{L^{p}(\mathbb{C}, \lambda)}^{p},
\end{aligned}
$$

so the lemma is proved.

Proposition 10. Let $p$ be a number with $1<p<\infty$. For any $f \in \mathcal{H}(\mathbb{C})$, if $w^{2} f \in L^{p}(\mathbb{C}, \lambda)$, then $f \in L^{p}(\mathbb{C}, \lambda)$. In particular, for any $n \in \mathbb{N}$ if $w^{2 n} f \in$ $\mathcal{H} L^{p}(\mathbb{C}, \lambda)^{e}$ then $f \in \mathcal{H} L^{p}(\mathbb{C}, \lambda)^{e}$.

Proof. Let $\mathbb{D}=\{w \in \mathbb{C}:|w| \leq 1\}$. Then $\|f\|_{L^{p}(\mathbb{C}-\mathbb{D}, \lambda)} \leq\left\|w^{2} f\right\|_{L^{p}(\mathbb{C}-\mathbb{D}, \lambda)}<$ $\infty$. Next, we will show that there is a constant $C>0$ such that

$$
\|f\|_{L^{p}(\mathbb{C}, \lambda)} \leq C\|f\|_{L^{p}(\mathbb{C}-\mathbb{D}, \lambda)} .
$$

Let $w \in \mathbb{D}$. Denote by $\mathcal{C}(w)$ the annulus $\{v \in \mathbb{C}: 2 \leq|v-w| \leq 3\}$. For any $v \in \mathcal{C}(w)$, we use the polar coordinates with the origin at $w$ so that $v-w=r e^{i \theta}$. Since $f \in \mathcal{H}(\mathbb{C})$, we expand $f$ as a power series around $v=w$ :

$$
f(v)=f(w)+\sum_{n=1}^{\infty} a_{n}(v-w)^{n} .
$$

Hence,

$$
\begin{aligned}
\int_{\mathcal{C}(w)} f(v) d v & =5 \pi f(w)+\sum_{n=1}^{\infty} \int_{2}^{3} \int_{0}^{2 \pi} a_{n} r^{n+1} e^{i \theta} d \theta d r \\
& =5 \pi f(w) .
\end{aligned}
$$

Thus

$$
\begin{aligned}
f(w) & =\frac{1}{5 \pi} \int_{\mathcal{C}(w)} f(v) d v \\
& =\frac{1}{5 \pi} \int_{\mathbb{C}-\mathbb{D}} \chi_{\mathcal{C}(w)}(v) \frac{1}{\Lambda(v)} f(v) \Lambda(v) d v .
\end{aligned}
$$

By Hölder's inequality we have that

$$
|f(w)| \leq \frac{1}{5 \pi}\left\|\chi_{\mathcal{C}(w)} \frac{1}{\Lambda}\right\|_{L^{q}(\mathbb{C}-\mathbb{D}, \lambda)}\|f\|_{L^{p}(\mathbb{C}-\mathbb{D}, \lambda)}
$$


where $\frac{1}{q}+\frac{1}{p}=1$. Since $\Lambda$ is strictly positive and continuous on $\mathcal{C}(w), \frac{1}{\Lambda}$ is bounded on $\mathcal{C}(w)$. Thus $\left\|\chi_{\mathcal{C}(w)} \frac{1}{\Lambda}\right\|_{L^{q}(\mathbb{C}-\mathbb{D}, \lambda)}$ is finite and

$$
\frac{1}{5 \pi}\left\|\chi_{\mathcal{C}(w)} \frac{1}{\Lambda}\right\|_{L^{q}(\mathbb{C}-\mathbb{D}, \lambda)} \leq \frac{1}{5 \pi}\left\|\chi_{\mathcal{C}^{*}} \frac{1}{\Lambda}\right\|_{L^{q}(\mathbb{C}-\mathbb{D}, \lambda)}<\infty
$$

where $\mathcal{C}^{*}=\{v \in \mathbb{C}: 1<|z|<4\}$ which $\mathcal{C}(w) \subset \mathcal{C}^{*}$ for all $w \in \mathbb{D}$. It follows that there exists a constant $c$ such that

$$
|f(w)| \leq c\|f\|_{L^{p}(\mathbb{C}-\mathbb{D}, \lambda)} .
$$

Hence

$$
\begin{aligned}
\int_{\mathbb{C}}|f(w)|^{p} d \lambda(w) & =\int_{\mathbb{D}}|f(w)|^{p} d \lambda(w)+\int_{\mathbb{C}-\mathbb{D}}|f(w)|^{p} d \lambda(w) \\
& \leq c^{p}\|f\|_{L^{p}(\mathbb{C}-\mathbb{D}, \lambda)}^{p} \lambda(\mathbb{D})+\|f\|_{L^{p}(\mathbb{C}-\mathbb{D}, \lambda)}^{p} \\
& \leq C\|f\|_{L^{p}(\mathbb{C}-\mathbb{D}, \lambda)}^{p},
\end{aligned}
$$

so the proposition is proved.

Corollary 11. Let $p$ be a number with $1<p<\infty$ and $F \in \mathcal{H}\left(\mathbb{C}^{d}\right)^{S O}(d, \mathbb{C})$. For any $n \in \mathbb{N}$ if $\mathcal{A}^{n}(F) \in \mathcal{H} L^{p}\left(\mathbb{C}^{d}, \mu\right)^{S O(d, \mathbb{C})}$ then $F \in \mathcal{H} L^{p}\left(\mathbb{C}^{d}, \mu\right)^{S O(d, \mathbb{C})}$.

Lemma 12. Let $p$ be a positive number with $1<p<\infty$ and $F \in$ $\mathcal{H}\left(\mathbb{C}^{d}\right)^{S O(d, \mathbb{C})}$. If there is $f \in L^{p}\left(\mathbb{R}^{d}, \rho\right)^{S O(d)}$ such that $B(f)=F$ and for all multi-indices $\alpha, \beta, \quad x^{\alpha}\left(\frac{\partial}{\partial x}\right)^{\beta} f(x) \in L^{p}\left(\mathbb{R}^{d}, \rho\right)$ then for any $n$ there is a constant $C_{n}$ such that

$$
|F(z)| \leq C_{n} e^{y^{2} / 2} e^{x^{2} / 2(p-1)} \frac{1}{\left(1+|(z, z)|^{n}\right)\left(1+|(z, z)|^{d-1}\right)}, \quad x+i y=(z, z) .
$$

Proof. From the proof of Theorem 7 of [7] tell us that for all positive integer $n_{i}, z_{i}^{n_{i}} F(z)$ is the Segal-Bargmann transform of some function in $L^{p}\left(\mathbb{R}^{d}, \rho\right)$. Thus $(z, z)^{n+d-1} F(z)=\left(z_{1}^{2}+z_{2}^{2}+\cdots+z_{d}^{2}\right)^{n+d-1} F(z)$ is also the Segal-Bargman transform of a function in $L^{p}\left(\mathbb{R}^{d}, \rho\right)^{S O(d)}$. By Theorem 2 of [7] there exists a constant $C_{n}$ such that

$$
|(z, z)|^{n+d-1} F(z) \mid \leq C_{n} e^{y^{2} / 2} e^{x^{2} / 2(p-1)} \quad x+i y=z .
$$

But the bilinear form $(\cdot, \cdot)$ and $F$ are $S O(d, \mathbb{C})$-invariant and

$$
|(z, z)|=\inf \left\{|A z|^{2}: A \in S O(d, \mathbb{C})\right\} .
$$


Therefore for all $z \in \mathbb{C}^{d}$,

$$
|F(z)| \leq C_{n} e^{y^{2} / 2} e^{x^{2} / 2(p-1)} \frac{1}{\left(1+|(z, z)|^{n}\right)\left(1+|(z, z)|^{d-1}\right)}
$$

where $x+i y=(z, z)$.

Theorem 13. Let $p$ be a positive number with $1<p<\infty$ and $F \in$ $\mathcal{H}\left(\mathbb{C}^{d}\right)^{S O(d, \mathbb{C})}$. If there is $f \in L^{p}\left(\mathbb{R}^{d}, \rho\right)^{S O(d)}$ such that $B(f)=F$ and for all multi-indices $\alpha, \beta, \quad x^{\alpha}\left(\frac{\partial}{\partial x}\right)^{\beta} f(x) \in L^{p}\left(\mathbb{R}^{d}, \rho\right)$ then $\mathcal{A}^{n}(F) \in \mathcal{H} L^{q}\left(\mathbb{C}^{d}, \mu\right)^{S O(d, \mathbb{C})}$ for all $n \in \mathbb{N}$ when $\frac{1}{p}+\frac{1}{q}=1,0<q \leq 2$ and $p \geq \frac{q}{2}+1$.

Proof. For any $n \in \mathbb{N}$, by Lemma 12 there is a constant $C_{n}$ such that

$$
|F(z)| \leq \frac{C_{n} e^{y^{2} / 2} e^{x^{2} / 2(p-1)}}{\left(1+|(z, z)|^{n}\right)\left(1+|(z, z)|^{(d-1)}\right)}, \quad x+i y=(z, z) .
$$

Following Lemma 7 we have that the $L^{q}$-norm with respect to $\lambda$ is equivalent to the $L^{q}$-norm with respect to the measure $\beta$. Then

$$
\begin{aligned}
\left\|\mathcal{A}^{n}(F)\right\|_{L^{q}\left(\mathbb{C}^{d}, \mu\right)}^{q} & =\int_{\mathbb{C}^{d}}\left|(z, z)^{n} F(z)\right|^{q} \mu(z) d z \\
& \leq \int_{\mathbb{C}^{d}}\left|(z, z)^{n}\right|^{q} \frac{C e^{q y^{2} / 2} e^{q x^{2} / 2(p-1)}}{\left(1+|(z, z)|^{n}\right)^{q}\left(1+|(z, z)|^{(d-1)}\right)^{q}} \mu(z) d z \\
& =\int_{\mathbb{C}}|w|^{2 n q} \frac{C e^{q y^{2} / 2} e^{q x^{2} / 2(p-1)}}{\left(1+|w|^{2(d-1)}\right)^{q}\left(1+|w|^{2 n}\right)^{q}} \lambda(w) d w \\
& \leq \int_{\mathbb{C}} K|w|^{2 n q} \frac{C e^{q y^{2} / 2} e^{q x^{2} / 2(p-1)}}{\left(1+|w|^{2(d-1)}\right)^{q}\left(1+|w|^{2 n}\right)^{q}} \frac{e^{-|w|^{2}}|w|^{d-1}}{\pi} d w
\end{aligned}
$$

Since $0<q \leq 2$ and $p \geq \frac{q}{2}+1, \frac{q}{2}-1 \leq 0$ and $\frac{q}{2(p-1)} \leq 0$. Thus $\left\|\mathcal{A}^{n}(F)\right\|_{L^{q}\left(\mathbb{C}^{d}, \mu\right)}^{q} \leq \int_{\mathbb{C}} K|w|^{2 n q} \frac{C e^{q y^{2} / 2} e^{q x^{2} / 2(p-1)}}{\left(1+|w|^{2(d-1)}\right)^{q}\left(1+|w|^{2 n}\right)^{q}} \frac{e^{-|w|^{2}}|w|^{d-1}}{\pi} d w<\infty$.

Hence for all $n \in \mathbb{N}, \mathcal{A}^{n}(F) \in \mathcal{H} L^{q}\left(\mathbb{C}^{d}, \mu\right)^{S O(d, \mathbb{C})}$. 


\section{Acknowledgments}

This research is supported by the Centre of Exellence in Mathematics, the Commission on Higher Education, Thailand.

\section{References}

[1] V. Bargmann, On a Hilbert space of analytic functions and an associated integral transform, Part I, Comm. Pure Appl. Math., 14 (1961), 187-214.

[2] G. Folland, Harmonic Analysis in Phase Space, Princeton University Press, Princeton, NJ (1989).

[3] L. Gross, P. Malliavin, Hall's transform and the Segal-Bargmann map, In: Ito's Stochastic calculus and Probability theory (Ed-s: M. Fukushima, N. Ikeda, S. Watanable) 73-116, Springer-Verlag, Berlin-New York, 1996.

[4] B.C. Hall, The Segal-Bargmann "coherent state" transform for compact Lie groups, J. Funct. Anal., 122 (1994), 103-151.

[5] B.C. Hall, Holomorphic methods in analysis and mathematical physics, In: First Summer School in Analysis and Mathematical Physics (Ed-s: S. Pèrez Esteva, C. Villegas Blas), 1-59, Contemp. Math., Vol. 260, Amer. Math. Soc., Providence, RI, 2000.

[6] B.C. Hall, Harmonic analysis with respect to heat kernel measure, Bull. Amer. Math. Soc. (N.S.), 38 (2001), 43-78.

[7] B.C. Hall, Bounds on the Segal-Bargmann transform of Lp functions, $J$. Fourier Anal. Appl., 7 (2001), 553-569.

[8] B.C. Hall, J.J. Mitchell, Coherent states on spheres, J. Math. Phys., 43 (2002), 1211-1236.

[9] A. Kaewthep, W. Lewkeeratiyutkul, A pointwise bound for rotationinvariant holomorphic functions that are square integrable with respect to a Gaussian measure, Taiwanese J. Math., 11 (2007), 1443-1455.

[10] A. Kaewthep, W. Lewkeeratiyutkul, Rotation-invariant Segal-Bargmann transform, East-West J. of Mathematics - A Special Valume (2007), 159167. 
[11] A. Kaewthep, W. Lewkeeratiyutkul, The characterization fo the rotationinvariant Segal-Bargmann space, International Jouranl of Pure and Applied Math., 61, No. 1 (2010), 43-52. 
